\title{
COSTECTOMY AS THE FIRST STAGE OF SURGERY FOR SCOLIOSIS
}

\author{
R. OWEN, A. TURNER, J. S. G. BAMFORTH, J. F. TAYLOR, R. S. JONES
}

From Alder Hey Children's Hospital, Liverpool

\begin{abstract}
Preliminary costectomy before Harrington instrumentation and fusion for idiopathic scoliosis allows direct excision of the rib prominence and better correction at the second-stage operation. The excised rib fragments are used as grafts, thus avoiding the need for a separate pelvic incision. The management regime and the technique of costectomy are described. The results in $\mathbf{4 2}$ children, most suffering from adolescent idiopathic scoliosis and all treated by this method, have been reviewed.

Respiratory function in a group of these children has been compared with that of a group treated by Harrington instrumentation alone. Costectomy produced a significantly greater reduction in total lung capacity and peak expiratory flow rate but, providing the preliminary lung function tests were reasonably normal, the cosmetic and psychological effects of costectomy were very rewarding.
\end{abstract}

The role of costectomy in the management of scoliosis has been controversial for many years. Most authorities consider that either a single-stage Harrington procedure with spinal fusion (Harrington 1962) or Luque posterior instrumentation is the treatment of choice in most cases of scoliosis which need operation.

However, some authors (Piggott 1971; Manning, Prime and Zorab 1973; Steel 1983) and ourselves have found that many patients, particularly adolescent girls, continue to complain about their rib prominence even after their scoliosis has been well corrected at operation (Figs 1 and 2). The rib hump remains as a psychological and cosmetic embarrassment. Some patients may also complain of neuralgic pain associated with the scar produced by taking bone grafts from the iliac crest.

Excision of the rib prominence before operation for Harrington instrumentation and fusion has been practised in Liverpool for some years; it has a number of advantages. First, direct excision of the rib prominence has a cosmetic advantage, and also allows better correction of the spinal curve at the second-stage operation. Second, the excised rib sections are used as grafts and the need for a separate incision over the iliac crest is avoided.

The disadvantages of preliminary costectomy are

R. Owen, MChOrth, FRCS, Professor of Orthopaedic and Accident Surgery

A. Turner, BSc, MCh Orth, FRCS, Senior Lecturer in Orthopaedic and Accident Surgery

University Department of Orthopaedic and Accident Surgery, Royal Liverpool Hospital, Prescot Street, Liverpool L7 8XP, England.

J. S. G. Bamforth, MRCP, Senior Registrar

Department of Medical Genetics, University Hospital of Wales, Heath Park, Cardiff CF4 4XW, Wales.

J. F. Taylor, MD, MChOrth, FRCS, Consultant Orthopaedic Surgeon R. S. Jones, MD, FRCP, Consultant Physician

Alder Hey Children's Hospital, Eaton Road, West Derby, Liverpool L12 2AP, England.

Requests for reprints should be sent to Mr A. Turner.

(C) 1986 British Editorial Society of Bone and Joint Surgery $0301-620 \mathrm{X} / 86 / 1019 \$ 2.00$ that two operations are required, and that rib excision can produce complications such as haemothorax, pneumothorax and intercostal neuralgia, although in practice these rarely occur. Respiratory function is also

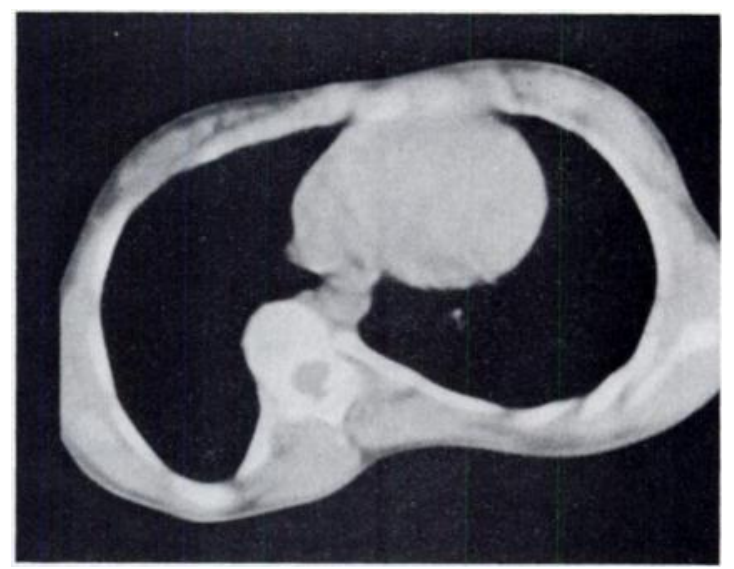

Fig. I

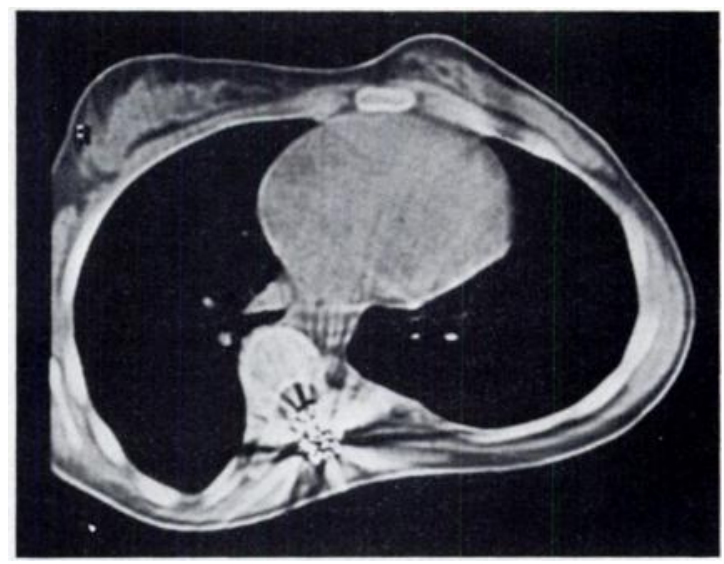

Fig. 2

CT scans at the level of the apex of a thoracic scoliosis before and after posterior instrumentation show that the rib prominence remains unchanged. 
affected; this is discussed later. A theoretical disadvantage is increased generalised osteoporosis, caused by the short additional period of recumbency necessitated by costectomy, and finally, the scar of the preliminary operation may be vulnerable to pressure from a localiser cast.

A number of patients who have been treated by preliminary costectomy, and by other methods in our Spinal Disorders Unit were reviewed in an attempt to define the indications, complications and results.

\section{PATIENTS AND METHODS}

Between August 1977 and December 1982 in this unit 42 children had preliminary costectomy a few weeks before Harrington instrumentation and posterior spinal fusion, and have been reviewed. There were 35 girls and 7 boys. Their mean age at presentation was 13 years 6 months (range 3 years to 17 years).

Table I. The aetiology of spinal curves in 42 patients

\begin{tabular}{ll} 
Type & Number \\
\cline { 2 - 2 } Idiopathic adolescent & 28 \\
Marfan's syndrome & 3 \\
Neurofibromatosis & 3 \\
Myelomeningocele & 2 \\
Congenital & 2 \\
Down`s syndrome & 1 \\
Cerebral palsy & 1 \\
Arthrogryposis & 1 \\
Infantile idiopathic & 1
\end{tabular}

The aetiology of the spinal curves is shown in Table I, 28 being of the adolescent idiopathic type. There were 37 single thoracic curves and 5 double curves; the mean Cobb angle of all the thoracic curves was $77^{\circ}$. Rib prominences were right-sided in 35 and left-sided in 7.

The mean age at costectomy was 14 years 2 months (range 6 years 6 months to 17 years 6 months). The mean number of ribs removed was 5 (range 4 to 6 ). The highest rib removed was the fourth; the lowest was the eleventh. Postoperatively 41 patients made a satisfactory recovery but one patient with severe heart disease secondary to Marfan's syndrome suffered severe impairment of cardiorespiratory function and was not fit for the second-stage operation. Instrumentation and fusion was therefore carried out on 41 of the patients. The mean extent of the fusion was 10 vertebrae, the highest level being $\mathrm{T} 3$ and the lowest $\mathrm{L} 4$.

Respiratory function. To assess the effect of preliminary costectomy on respiratory function, 21 patients ( 17 girls and 4 boys) from the total series of 42 who had a preliminary costectomy before Harrington instrumentation were compared with 16 patients ( 15 girls and 1 boy) who had Harrington instrumentation as the only procedure in the same unit.

The two groups of patients had similar ranges of age and of arm span, and a similar correction of the Cobb angle was achieved at operation. The total lung capacity, vital capacity, residual volume, peak expiratory flow rate and forced expiratory volume in one second were measured in all patients in both groups, one week before treatment and again one year postoperatively. These parameters of lung volume were measured by a helium dilution technique and the results were expressed as a percentage of that value predicted for normal individuals with the same arm span (Polgar and Promadhat 1971). The results were analysed using the paired Student's $t$-test.

Regime for preliminary costectomy. A full assessment was carried out including measurement of spinal curves, clinical photography and respiratory function tests. Costectomy was performed as detailed below and, when the wound had healed, usually in about two weeks, operation for Harrington instrumentation and posterior spinal fusion was performed, using the rib segments as grafts. A well-fitting localiser cast was applied when the wound had healed, and the patient was then allowed up. In the early years of this study, the cast was maintained for 12 months; but we now believe that improved techniques of fixation allow earlier mobilisation out of the cast. Further clinical assessment, photography and respiratory function tests were performed, and 18 months after the fusion operation a technetium bone scan was performed to exclude pseudarthrosis.

Technique of costectomy. The patient is placed prone on a Toronto frame and, in the line of the incision, the skin is infiltrated with adrenaline 1:500000 in saline. The incision is an oblique one along Langer's lines over the apex of the rib prominence, rather than parallel to the vertically inclined ribs. The medial end of the incision does not reach the midline, so that the scar will not reach the subsequent incision for spinal fusion. This avoids the danger that the triangular flap of skin between the two incisions will become ischaemic. The lateral end reaches the posterior axillary line. Subcutaneous tissue and muscle are divided with cutting diathermy down to bone. Ribs are exposed subperiosteally on either side of the apex of the prominence and a section of each prominent rib is excised from the posterior axillary line laterally to the neck of the rib medially, deliberately leaving the head of the rib in situ. The uppermost and lowest ribs need not be excised as widely. The costotransverse joint is included in the excision but the transverse processes are dealt with during the second-stage procedure. Skin retraction allows the necessary number of ribs to be removed through one incision. The rib cage is then stabilised with strong braided nylon sutures passed above and below the intact remaining ribs proximally and distally, which are then approximated as closely as possible by tying the sutures under tension. An inter- 
Table II. Span, size of curve and respiratory function tests in 16 patients undergoing Harrington procedure alone, given as mean $\pm 1 S D$

\begin{tabular}{|c|c|c|c|c|}
\hline & Pre-operative & Postoperative & Change & $\begin{array}{l}\text { Significance } \\
\text { of change }(P)\end{array}$ \\
\hline Span $(c m)$ & $160 \pm 10.7$ & $165 \pm 9.1$ & +5 & $0.005+$ \\
\hline Cobb angle (degrees) & $59.6 \pm 10$ & $35.9 \pm 13$ & -23.7 & $<0.001 \dagger$ \\
\hline Total lung capacity* & $75.6 \pm 8.7$ & $66.6 \pm 10$ & -9 & 0.15 \\
\hline Vital capacity* & $74.9 \pm 17$ & $63.3 \pm 15$ & -11.6 & $0.05+$ \\
\hline Forced expiratory volume in one second & $79.8 \pm 14$ & $65.8 \pm 13$ & -14 & $0.01+$ \\
\hline Peak expiratory flow rate & $83.7 \pm 12$ & $84.3 \pm 14$ & -0.6 & $>0.1$ \\
\hline Residual volume & $106 \pm 39$ & $108 \pm 22.2$ & +2 & $>0.1$ \\
\hline
\end{tabular}

* Results of lung function tests are expressed as a percentage of that predicted for normal patients with the same span $+P$ values of 0.05 and less are regarded as significant

Table III. Span, size of curve and respiratory function tests in 21 patients undergoing costectomy followed by the Harrington procedure

$\begin{array}{lcccc} & \text { Pre-operative } & \text { Postoperative } & \text { Change } & \begin{array}{c}\text { Significance } \\ \text { of change }(\boldsymbol{P})\end{array} \\ \text { Span }(\mathrm{cm}) & 157.2 \pm 3.9 & 163.3 \pm 11.7 & +6.1 & 0.025+ \\ \text { Cobb angle (degrees) } & 67.8 \pm 17.8 & 44.25 \pm 13 & -23.55 & <0.001+ \\ \text { Total lung capacity* } & 70.3 \pm 13.8 & 56.2 \pm 10.6 & -14.1 & 0.001+ \\ \text { Vital capacity* } & 64.8 \pm 15.1 & 48.2 \pm 9 & -16.6 & 0.001+ \\ \text { Forced expiratory volume in one second } & 71.9 \pm 5.5 & 54.3 \pm 13.3 & -17.6 & <0.001+ \\ \text { Peak expiratory flow rate* } & 88.7 \pm 16.6 & 81.9 \pm 3.8 & -6.8 & 0.01+ \\ \text { Residual volume* } & 92.3 \pm 29 & 81.1 \pm 27 & -11.2 & >0.1\end{array}$

* Results of lung function tests are expressed as a percentage of that predicted for normal patients with the same span $+P$ values of 0.05 and less are regarded as significant

costal drain is inserted and also a closed suction wound drain.

The wound is closed with a continuous absorbable subcuticular stitch. The excised ribs are cut into chips and stored for subsequent use. Postoperatively the child is encouraged to lie tilted to the side of rib removal. The second stage is performed when the costectomy wound has healed, usually after about two weeks. During the period between operations Cotrel intermittent dynamic traction is given.

\section{RESULTS}

Of the 41 patients undergoing the spinal fusion stage of treatment, 40 had no postoperative complications, but one developed superior mesenteric artery syndrome with duodenal obstruction. This settled satisfactorily with conservative management. The mean curve for the whole group before treatment was 77 ; after costectomy and Harrington instrumentation it was $36^{\circ}$, giving a mean early correction of 41 . One year after the two-stage operation the mean curve was 45 , a loss of correction of 9 in one year, leaving a r.can correction of 32 at late review.
Respiratory function tests were carried out on two matched groups of patients. Table II gives the mean results for 16 patients who had a Harrington procedure and iliac graft fusion; Table III gives the results for 21 of the patients who had a preliminary costectomy before their Harrington procedure and rib graft.

\section{DISCUSSION}

Roaf (1966) and Manning et al. (1973) have reported the use of costectomy as a cosmetic procedure, after a spinal fusion and at the end of growth. Steel (1983) reported performing convex rib excision at the same time as spinal fusion in 370 patients and in a further 22 patients he excised rib humps at a later date when the spinal fusion was solid. His method of costectomy differs from that reported by us in that it is usually done at the same time as the spinal fusion and is undertaken through a separate vertical incision over the rib prominence. The most serious complication he reported was haemothorax which occurred before he made routine use of intrapleural drainage. He checked carefully for perforation of the pleura at the end of the operation by flooding the wound with saline and watching for bubbles. He then 


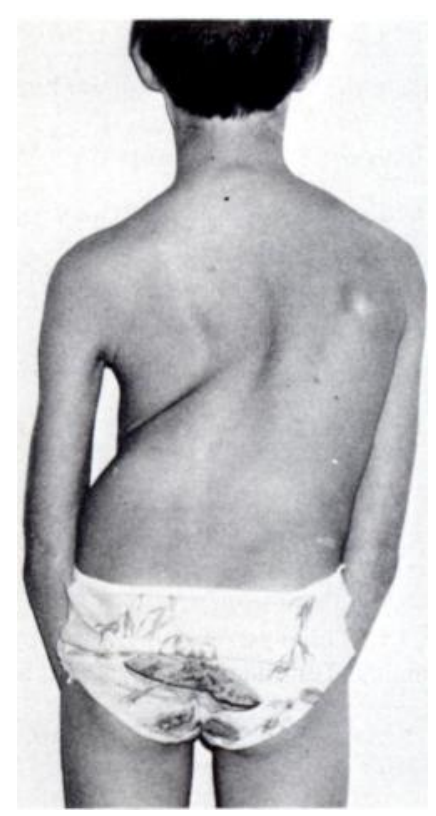

Fig. 3

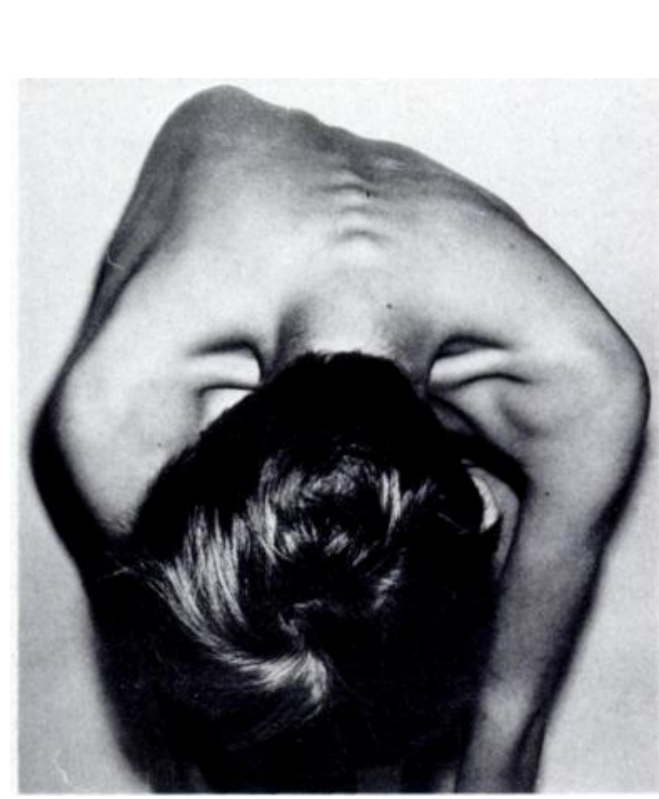

Fig. 4

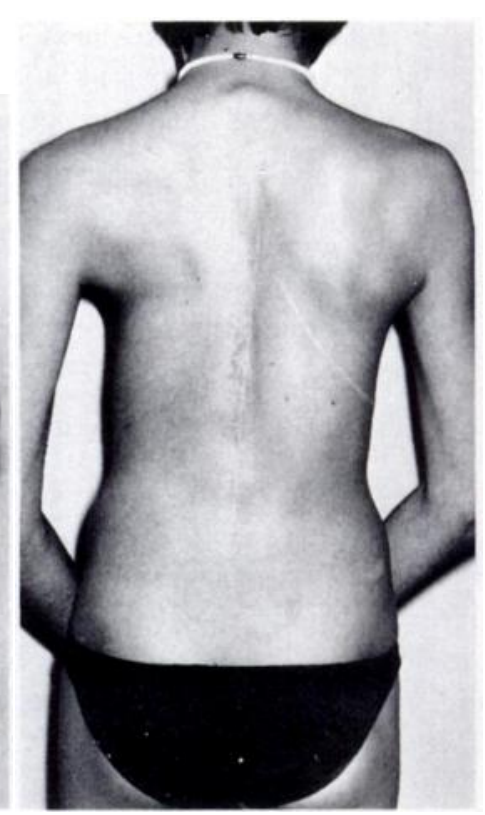

Fig. 5

Figures 3 and 4 -Photographs of a 12-year-old girl with an idiopathic right thoracic scoliosis and right rib prominence. Figure 5 - After a two-stage procedure there is excellent correction of both the curve and the rib prominence.

repaired any defect, theoretically negating the need for intrapleural drainage. We have routinely inserted a chest drain and have had no complications from haemothorax or pneumothorax. Steel reported that the excised ribs reformed in their new position after about three months and we have also found this to occur.

Steel reported unsightly protuberance of the medial stumps of the resected ribs in two patients, which caused discomfort when the patient sat on a hard-backed chair. James (1976) also thought that this might cause difficulty but we have not had similar problems in our series. One of our patients required cosmetic excision of the inferior pole of the scapula at the end of growth; the need for this has been noted by other authors (Roaf 1980; Steel 1983). We try to avoid this procedure, preferring instead to remove the angle of the underlying rib; this can be done at the preliminary costectomy.

The tests of respiratory function reported in Tables II and III show that patients undergoing Harrington instrumentation alone have significant reductions only in vital capacity and forced expiratory volume in one second, whereas those who have preliminary costectomy followed by Harrington instrumentation have a significant reduction in these measurements and also in total lung capacity and peak expiratory flow rate.

Progressive scoliosis leads to increasing deformity of the thoracic cage and this results in reduced intrathoracic volume and decreased chest wall compliance (Bergofsky 1979). These factors lead to alveolar hypoventilation and subsequent cardiorespiratory embarrassment (Bergofsky 1967). Patients with Cobb angles of more than 70 are particularly at risk (Caro and DuBois 1961). Westgate and Moe (1969) and Shannon, Riseborough and Kazemi (1971) have shown that Harrington instrumentation alone, although improving the Cobb angle and spinal stability, does little to improve lung volumes; this is confirmed by our results (Table II).

Costectomy prior to Harrington instrumentation probably results in the loss of some functional lung tissue beneath the rib prominence and also in a reduction in the total volume of the thoracic cage. This is apparent in the relatively greater reduction in some parameters of lung function in patients undergoing preliminary costectomy.

A significant reduction in the peak expiratory flow rate following preliminary costectomy and Harrington instrumentation may arise as a result of the initially smaller vital capacity seen in these patients as compared with those undergoing Harrington instrumentation alone. Disruption of the intercostal muscles in the region of the costectomy may also play a part. It is interesting that Steel (1983) found a reduction in pulmonary function in the first two weeks after combined costectomy and spinal fusion. However, these measurements gradually improved with time, until three years after surgery all patients had pulmonary function the same as, or better than, that before operation. This may, however, have been due to growth of the thoracic cage rather than to any functional improvement.

In the light of our respiratory function studies, therefore, we believe that patients with severely restricted pre-operative lung volumes should not undergo costectomy as a preliminary to Harrington instrumentation because the effect on respiratory function will be correspondingly greater. This is well illustrated by one patient in our series, a boy with Marfan's syndrome and severe congenital heart disease. After much deliberation he was subjected to preliminary cosctomy, and this must be regarded as a failure because it resulted in cardiorespira- 
tory deterioration of such an extent that the second stage of correction and fusion could not be carried out.

In conclusion, our policy now is to reserve preliminary costectomy almost entirely for patients with idiopathic curves although occasionally it may be used for deformities of known aetiology. The excellent cosmetic results obtainable by this two-stage procedure are shown in Figures 3 to 5. Preliminary costectomy is of course applicable only to the management of thoracic curves, being of no value for curves which are mainly lumbar. Pre-operative lung function tests should have given reasonable results. When rib prominence is mild or minimal, then single-stage Harrington or Luque instrumentation is the treatment of choice. Modern midline instrumentation can produce some derotation of the apical thoracic vertebral bodies but, in a patient with a rigid thoracic cage, derotation alone may leave the patient with a not inconsiderable prominence of the type shown in Figure 2.

We are grateful to $\mathrm{Mr}$ J. Dove, FRCS, of the Stoke Spinal Unit, for allowing us to use CT scans of his patients, and we would also like to thank Mrs G. Berry for typing the manuscript.

\section{REFERENCES}

Bergofsky EH. Cor pulmonale in the syndrome of alveolar hypoventilation. Prog Cardiovasc Dis 1967:9:414-37.

Bergofsky EH. Respiratory failure in disorders of the thoracic cage. $\mathrm{Am}$ Rev Respir Dis 1979;119:643 69.

Caro CG, DuBois AB. Pulmonary function in kyphoscoliosis. Thorax $1961 ; 16: 282-90$.

Harrington PR. Treatment of scoliosis: correction and internal fixation by spine instrumentation. J Bone Joint Surg $[\mathrm{Am}]$ 1962;44-A: $591-610$.

James JIP. Scoliosis, 2nd ed. Edinburgh etc: Churchill Livingstone, 1976:330-1.

Manning CW, Prime FJ, Zorab PA. Partial costectomy as a cosmetic operation in scoliosis. J Bone Joint Surg [Br] 1973;55-B:521-7.

Piggott H. Posterior rib resection in scoliosis: a preliminary report. J Bone Joint Surg [Br] 1971;53-B:663-71.

Polgar G, Promadhat V. Pulmonary function testing in children: techniques and standards. Philadelphia and London: WB Saunders, 1971.

Roaf R. Scoliosis. Edinburgh: E \& S Livingstone, 1966:128-32.

Roaf R. Spinal deformities. 2nd ed. Tunbridge Wells: Pitman Medical, 1980:341.

Shannon DC, Riseborough EJ, Kazemi H. Ventilation perfusion relationships following correction of kyphoscoliosis. JAMA 1971: 217:579-84.

Steel HH. Rib resection and spine fusion in correction of convex deformity in scoliosis. J Bone Joint Surg [Am] 1983;65-A:920-5.

Westgate HD, Moe JH. Pulmonary function in kyphoscoliosis before and after correction by Harrington instrumentation method. J Bone Joint Surg [Am] 1969;51-A:935-46. 